\title{
Rectangular Microstrip Antenna with Air Cavity for High Gain and Improved Front to Back Ratio
}

\author{
Kaushik Malakar, Jaydeep Nandi, Souvik Mitra, Prasanta Kumar Gorai, Sudipta Chattopadhyay, \\ Sandip Banerjee
}

Department of ECE, Siliguri Institute of Technology, Siliguri, Darjeeling, India.

Email: piyalirekha@yahoo.com

Received July $2^{\text {nd }}, 2011$ revised July $29^{\text {th }}, 2011$; accepted August $12^{\text {th }}, 2011$.

\begin{abstract}
A rectangular microstrip patch antenna using conventional Poly Tetra Fluride Ethelene (PTFE) substrate with air cavity is proposed and theoretically investigated. Considerably high gain along with improved front to back radiation isolation is demonstrated using such proposed antenna. The radiation performance of this new antenna has been compared to a conventional microstrip patch for some commonly used aspect ratios (width to length ratio). Compared to conventional microstrip antenna the proposed configuration shows more than $12 \%$ increment in peak gain and more than $10 \%$ increment in front to back radiation performance in each set of aspect ratio. The elucidation of such improvement in the radiation characteristics of the proposed antenna is also presented.
\end{abstract}

Keywords: Air Cavity, Front to Back Radiation, High Gain, Microstrip Antenna

\section{Introduction}

Microstrip patch of rectangular or circular geometry etched on a grounded substrate is well known as printed antenna radiating along the broad side. Such a microstrip patch antenna on high dielectric constant substrate suffers from poor radiation pattern due to its high surface wave loss. But, sometimes in case of low dimensional devices, it is preferred to use a material with high dielectric constant in order to prevent spurious radiation. In those cases, microstrip patch when etched on a high dielectric constant substrate, show poor gain. In order to alleviate the lacunae, a microstrip patch antenna on PTFE-air mixed substrate configuration is chosen as a substrate for the proposed antenna in such a way that, the overall radiation performance can be improved. Several methods have been reported to minimize the surface wave losses such as complete or partial etching of substrate underneath the antenna [1-4] or developing few holes in the substrate to perturb the formation of substrate modes $[5,6]$. One very recently reported article on microstrip antenna with composite substrate configuration by one of the present authors, show high gain broad beam radiation pattern [7]. Some other composite substrate configurations [8] have been reported to enhance the gain of an antenna. But none of the above literatures reported the performance of their proposed antenna from the view of front to back radiation isolation.

Actually, the widely used conventional microstrip patch antenna with PTFE substrate produces low gain and poor front to back radiation characteristics. With a view to develop an antenna with high gain along with improved front to back ratio, we have proposed a simple rectangular microstrip patch with full air cavity beneath the antenna. This is done to reduce the effective synthesized dielectric constant below the patch and as a result to increase the peak gain along with improved front to back radiation performance. Larger gain is always a positive requirement but along with that if the front to back radiation isolation can be improved, it will be a promising feature for an antenna to be used as an efficient feed of reflector or minimizing interference in wireless mobile communication systems. In this new antenna, though the air becomes a part of the composite dielectric used, shown in Figure 1, it is still compatible with an integrated circuits and at the same time enjoys the gain enhancing feature as is done using simple air substrate below a patch along with improved front to back ratio.

The design and implementation of the antenna is simple. An electromagnetic simulator [9] has been used to study the characteristics theoretically and also to search for the physical insight in to this. Its radiation characteristics have been theoretically studied using a set of pro 


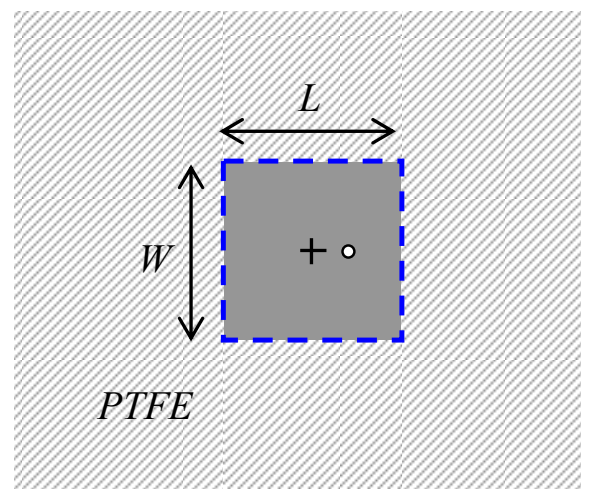

(a)

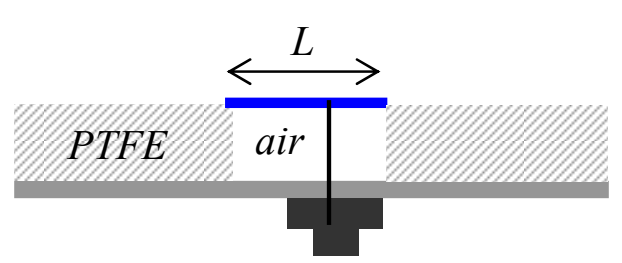

(b)

Figure 1. Rectangular Microstrip Antenna with Air Cavity. (a) Top View; (b) Cross sectional view.

totype and those results are compared. The increment of the gain and improvement in front to back ratio compared to those due to an identical patch on conventional PTFE substrate is theoretically demonstrated. The effect of varying aspect ratio $(W / L)$ on the radiation performance of such proposed antenna is also presented.

\section{Antenna Structure}

A simple rectangular microstrip antenna of length $L=20$ $\mathrm{mm}$ with a full air cavity beneath the patch and rest part filled with simple PTFE substrate has been designed. The top and cross sectional view of the proposed antenna has been shown in Figures 1(a) and (b) respectively. Three sets of such antenna structures with some commonly used aspect ratios i.e., $W / L \approx 0.8,1.0$ and 1.5 keeping its length fixed ( $L=20 \mathrm{~mm}$ ) has been designed using [9] to operate around C-band. A ground plane measuring $1.5 \lambda_{0}$ has been used for this present study. Taconic's TLY-3-0620 $\left(\varepsilon_{r}=\right.$ 2.33) with thickness $h=1.575 \mathrm{~mm}$ has been used as the PTFE substrate. The feed location has been optimized based on good matching for either configuration.

\section{Results}

The comparison of radiation characteristics of the proposed antenna with conventional microstrip patch antenna is shown in Figure 2 for different aspect ratios. A comprehensive result is presented in tabular form in Table 1 for clear indication of the effects. Figure 2 and Table 1 indicate an increase in peak gain with the increase in aspect ratio values and this is consistent for all the configurations. It is also evident that the proposed antenna result in higher gain than a conventional patch. About $1.8 \mathrm{~dB}$ improvement in peak gain value is observed for $W / L=0.8$. Moreover, the antennas with $W / L=$ 0.8 result in significantly improved front to back ratio if compared to an identical conventional patch. Similar observations like increment of peak gain values and improvement in front to back radiation ratio compared to identical conventional microstrip patch antenna are equally valid for the patches with other $W / L$ values like $W / L=1$ or 1.5 . It would be relevant to note that the proposed antenna produce larger side lobe levels as shown in Figure 2.

\section{Theoretical Analysis and Discussions}

At first we may concentrate on the reason of increment in peak gain values with aspect ratio. If a micristrip patch of length $L$ and width $W$ is fed with strip line or probe, the dominant mode is excited and the concept of fringing length $(\Delta L)$ and width $(\Delta W)$ comes. The effective radiating length $L_{g}$ and width $W_{g}$ comes as (Figure 3)

$$
\begin{gathered}
L_{g}=L+2 \Delta L \\
W_{g}=W+2 \Delta W
\end{gathered}
$$

The fringing length $\Delta L$, incorporating the effect of $\Delta W$ comes as [10].

$$
\Delta L=\pi a[\sqrt{(1+q)}-1] / 2[2.5-0.5(W / L)]
$$

where $a=W / 1.44$ and $q$ is the fringing factor as obtained from [11].

Now as we increase $W / L$ the fringing length $\Delta L$ increases and the effective aperture of the radiating antenna increases as

$$
A_{\text {eff }}=L_{g} W_{g}=(L+2 \Delta L)(W+2 \Delta W)
$$

hence the gain $G \propto A_{\text {eff }} / \lambda_{0}^{2}$ increases.

Now, following the concept of [12], when we have made an air cavity below the physical area of the patch the synthesized dielectric constant $\left(\varepsilon_{r s y n t h}\right)$ becomes proportional to the volume of the substrate removed underneath the microstrip antenna. This is because of the fact that the dielectric constant is basically a capacitance associated with unit volume (i.e. capacitance of two plates having unit area and separated by unit distance). Thus due to the development of air cavity $(L \times W)$ within the substrate and below the effective radiating area $\left(L_{g} \times\right.$ $W_{g}$ ) below the patch, the capacitance change reflects the patch, the capacitance change reflects the change in resultant synthesized dielectric constant which may be calculated as follows:

If, $C_{1}=$ capacitances of region $\mathrm{ABCD}, C_{2}=$ capaci- 
Table 1. Comparison of Radiation Characteristics for Patches with Conventional and Composite Substrates (proposed) having different aspect ratio: Substrate Thickness $=1.575 \mathrm{~mm}$.

\begin{tabular}{ccccc}
\hline \multirow{2}{*}{ Patch Description } & \multicolumn{2}{c}{ Conventional Patch } & \multicolumn{2}{c}{ Microstrip Patch with Air Cavity } \\
\cline { 2 - 5 } & Peak Gain (dBi) & F/B radiation isolation $(\mathrm{dB})$ & Peak Gain (dBi) & F/B radiation isolation (dB) \\
\hline$W / L=0.8 ; L=20$ & 7.0 & 14 & 8.79 & 21 \\
$W / L=1 ; L=20$ & 7.9 & 14 & 8.93 & 21 \\
$W / L=1.5 ; L=20$ & 8.2 & 17 & 9.57 & 19 \\
\hline
\end{tabular}
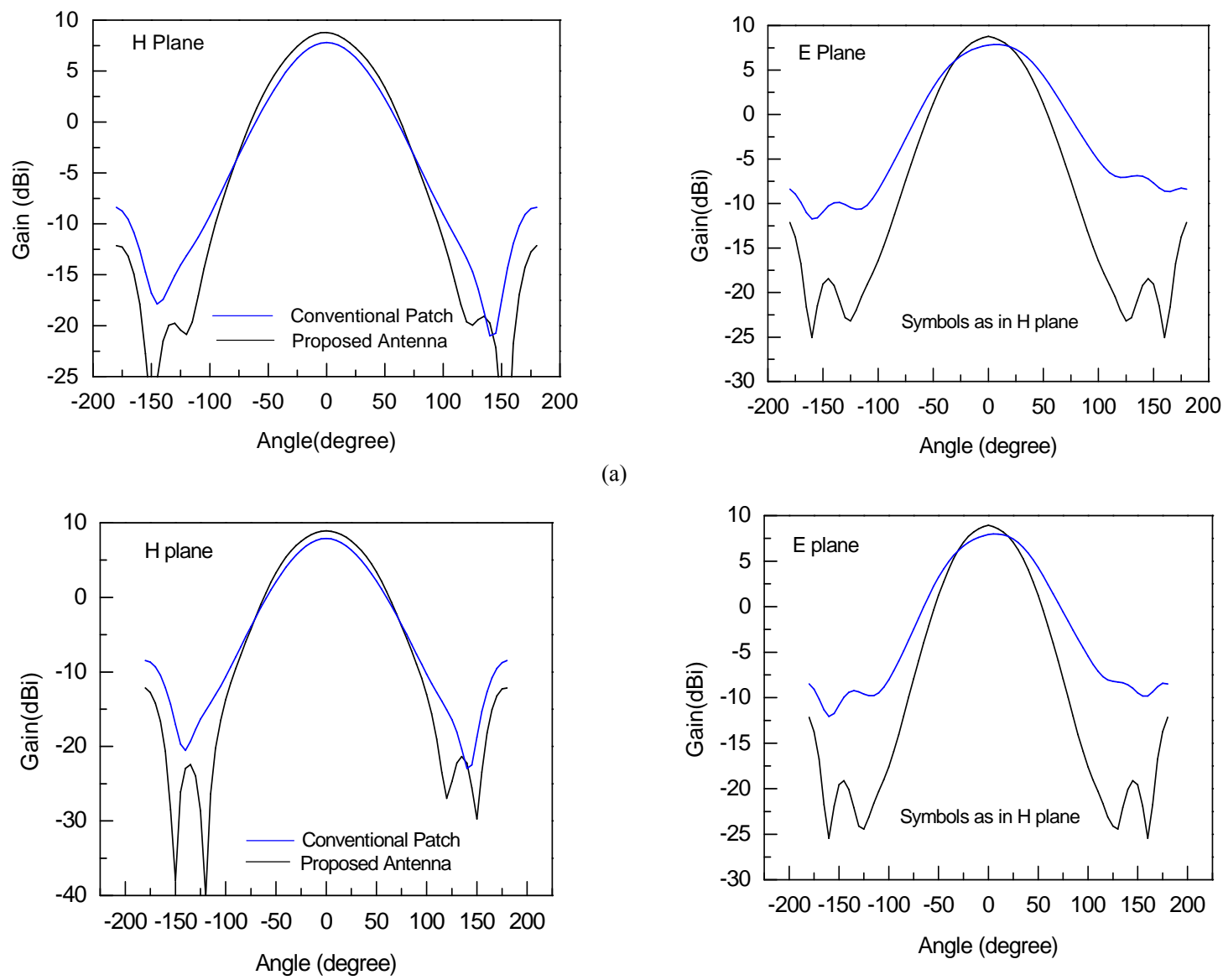

(b)
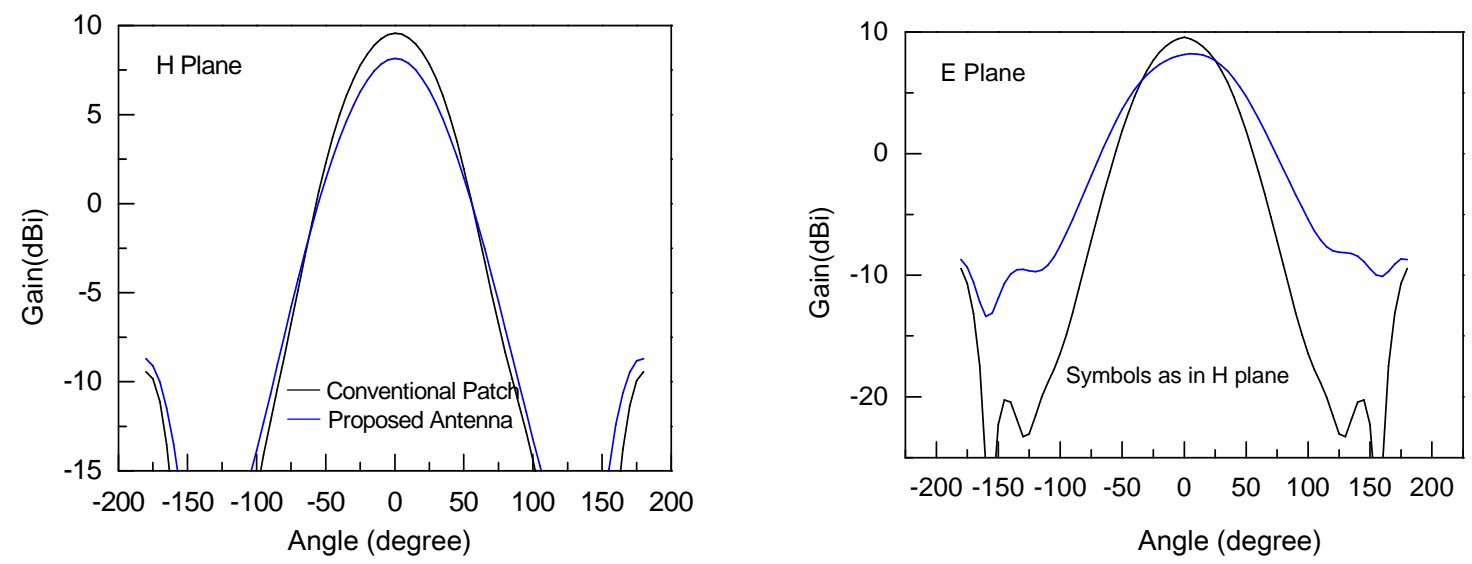

(c)

Figure 2. Radiation patterns compared for proposed antenna with conventional Microstrip antenna. $L=20 \mathrm{~mm}, h=1.575$ $\mathrm{mm}, \varepsilon_{r}=2.33$ (PTFE) and 1(air). (a) $W / L=0.8$; (b) $W / L=1.0$; (c) $W / L=1.5$. 
tances of regions EFGDAH and MBCQPN, $C_{3}=$ equivalent capacitances of regions DGQC and HABM in Figure 3.

Then,

$$
\begin{gathered}
C_{1}=\varepsilon_{\text {air }} \cdot W L / h ; \quad \varepsilon_{\text {air }}=1 \\
C_{2}=\varepsilon_{r} \cdot\left(W_{g}-W\right) L_{g} / h \\
C_{3}=\varepsilon_{r} \cdot\left(L_{g}-L\right) W / h
\end{gathered}
$$

Hence the effective capacitance $\left(C_{\text {eff }}\right)$ below the effectively radiating area,

$$
\begin{aligned}
& C_{e f f}=C_{1}+C_{2}+C_{3} \\
& \varepsilon_{r s y n t h} \cdot L_{g} W_{g} / h \\
& =\varepsilon_{\text {air }} \cdot W L / h+\varepsilon_{r} \cdot\left(W_{g}-W\right) L_{g} / h+\varepsilon_{r} \cdot\left(L_{g}-L\right) W / h
\end{aligned}
$$

Dividing both the sides by $L_{g} W_{g} / h$;

$$
\varepsilon_{\text {rsynth }}=\varepsilon_{\text {air }} \cdot W L / W_{g} L_{g}+\varepsilon_{r}-\varepsilon_{r} \cdot W L / W_{g} L_{g}
$$

Thus,

$$
\varepsilon_{r s y n t h}=\varepsilon_{r}(1-r)+r
$$

where $r=W L / W_{g} L_{g}$ is the proportion of volume removed.

As we know, $L_{g} \triangleright L$ and $W_{g} \triangleright W$, so $r \triangleleft 1$ always; which will lower the resultant $\varepsilon_{\text {rsynth }}$.

Hence, the fields are loosely bound within the antenna near field region and they will contribute for radiation in the broadside region. This may be attributed for higher peak gain value for the proposed antenna compared to conventional patch antenna for each set of aspect ratio. as the air content between the patch and ground plane increases, with larger dimension of patch (larger $W / L$ values) the resultant synthesized dielectric constant $\varepsilon_{r s y n t h}$

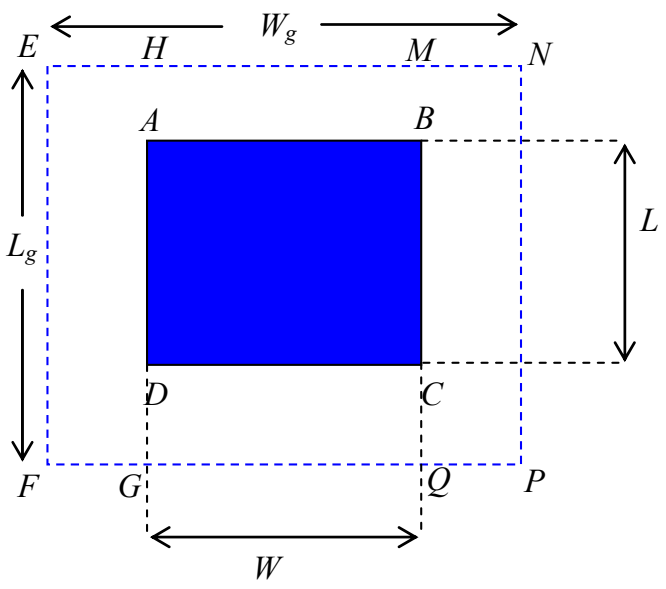

Figure 3. Top view of the Microstrip patch (Physical aperture and effective radiating aperture including fringing length and width). reduces more. Hence the fields are loosely bound below the patch and as a result, the peak gain of the antenna increases gradually. But when the fields are far from the patch periphery, they have become trapped by high dielectric constant material. This in fact reduces the possibility of radiation in the back side of antenna due to diffraction from edge of ground plane.

As we know, reflection coefficient $(\Gamma)$,

$$
\Gamma=\eta_{2}-\eta_{1} / \eta_{2}+\eta_{1}
$$

Again, $\eta \propto 1 / \sqrt{\varepsilon_{r s y n t h}}$

where, $\eta_{1}$ is intrinsic impedance of composite substrate material and $\eta_{2}$ is intrinsic impedance of ground plane which is having nearly zero value.

As we have introduced air below the patch, resultant synthesized dielectric constant $\varepsilon_{r s y n t h}$ decreases thereby increasing the value of $\eta_{1}$. Thus reflection coefficient $\Gamma$ increases. As a result, the possibility of radiation leaked through ground plane or transmission at back side reduces and hence improve the front to back radiation isolation characteristic for the proposed antenna compared to conventional one. But as the aspect ratio $W / L$ of the patch increases, fringing fields will be distributed over wider region as $\Delta L$ and $\Delta W$ increases with $W / L$ as indicated earlier. Thus the possibility of radiation in the back side of the antenna due to the edge diffraction effect increases and this may be attributed for degradation of front to back radiation isolation characteristic of the proposed structure. It should be relevant to note that, for highest optimum $W / L$ value $(W / L=1.5)$, the front to back radiation isolation is still $2 \mathrm{~dB}$ larger compared to conventional patch antenna.

\section{Conclusions}

A simple rectangular microstrip antenna with air cavity is proposed for high gain and improved front to back radiation characteristics. The proposed antenna is very helpful for antenna community and especially for antenna designers who are looking for wireless antennas with better radiation performance with less interference. There should be a plenty of scopes and opportunities for incorporating the concept in other patch geometries. Those may include studying with PTFE of different higher permittivity values and also, employing other possible composite configurations to explore several new radiation characteristics.

\section{REFERENCES}

[1] D. R. Jackson, J. T. Williams and A. K. Bhattacharyya, "Microstrip Patch Designs That do Not Excite Surface Waves," IEEE Transactions on Antennas and Propagation, Vol. 41, No. 8, 1993, pp. 1026-1037. 


\section{doi: $10.1109 / 8.244643$}

[2] W. Y. Ali-Ahmad, W. L. Bishop and T. W. Growe, "An 86-106 GHz Quasi-Integrated Low Noise Schottky Receiver," IEEE Transactions on Microwave Theory and Techniques, Vol. 41, No. 4, 1993, pp. 558-564.

[3] M. Slotz, G. Gottwald, H. Haspeklo and J. Wenger, "Planar Single- and Dual-Polarized Aperture Coupled E-Band Antennas on GaAs Using $\mathrm{SiN}_{\mathrm{x}}$-Membranes," Antennas and Propagation Society International Symposium, Baltimore, 21-26 July 1996, pp. 1540-1543.

[4] R. F. Drayton, "The Development and Characterization of Self-Packages Using Micromachining Techniques for High Frequency Circuit Applications," PhD Thesis, University of Michigan, Ann Arbor, 1995, pp. 102-123.

[5] M. J. Vaughan, K. Y. Hur and R. C. Compton, "Improvement of Microstrip Patch Antenna Radiating Patterns," IEEE Transactions on Antennas Propagation, Vol. 42, No. 6, 1994, pp. 882-885. doi:10.1109/8.301717

[6] G. V. Elefttheriades, "Analysis and Design of Integrated-Circuit Horn Antennas for Millimeter and Submilimeter-Wave Applications," PhD Thesis, University of Michigan, Ann Arbor, 1993, pp. 151-178.

[7] S. Chattopadhyay, J. Y. Siddiqui and D. Guha, "Rectangular Microstrip Patch on a Composite Dielectric Sub- strate for High Gain Wide Beam Radiation Patterns," IEEE Transactions on Antennas \& Propagations, Vol. 57, No. 10, 2009, pp. 3324-3327. doi:10.1109/TAP.2009.2029607

[8] S. Chattopadhyay, J. Y. Siddiqui and D. Guha, "Rectangular Patch on Air and Air-Dielectric Composite Substrates to Achieve Improved Radiation Characteristics," Proceedings of Applied Electromagnetics Conference, Kolkata, 14-16 December 2009, pp. 1-4.

[9] High Frequency Structure Simulator, v. 10.

[10] S. Chattopadhyay, M. Biswas, J. Y. Siddiqui and D. Guha, "Input Impedance of Probe-Fed Rectangular Microstrip Antennas with Variable Air-Gap and Varying Aspect Ratio," IEE Microwaves, Antennas \& Propagation, Vol. 3, No. 8, 2009, pp.1151-1156.

[11] S. Chattopadhyay, M. Biswas, J. Y. Siddiqui and D. Guha, "Rectangular Microstrips with Variable Air-Gap Varying Aspect Ratio: Improved Formulation and Experiments," Microwave Optical Technology Letters, Vol. 51, No. 1, 2009, pp. 169-173.

[12] G. P. Gauthier, A. Courtay and G. M. Rebeiz, "Microstrip Antennas on Synthesized Low Dielectric Constant Substrate," IEEE Transactions on Antennas and Propagation, Vol. 45, No. 8, 1997, pp. 1310-1314. 